\title{
Blue straggler stars in Galactic open clusters and the effect of field star contamination (Research Note)
}

\author{
G. Carraro ${ }^{1}$, R. A. Vázquez ${ }^{2}$, and A. Moitinho ${ }^{3}$ \\ 1 ESO, Alonso de Cordova 3107, Vitacura, Santiago, Chile* \\ e-mail: gcarraro@eso.org \\ 2 Facultad de Ciencias Astronómicas y Geofísicas de la UNLP, IALP-CONICET, Paseo del Bosque s/n, La Plata, Argentina \\ e-mail: rvazquez@fcaglp.unlp.edu.ar \\ 3 SIM/IDL, Faculdade de Ciências da Universidade de Lisboa, Ed. C8, Campo Grande, 1749-016 Lisboa, Portugal \\ e-mail: andre@sim.ul.pt
}

Received 7 September 2007 / Accepted 19 February 2008

\begin{abstract}
Context. We investigate the distribution of blue straggler stars in the field of three open star clusters.

Aims. The main purpose is to highlight the crucial role played by general Galactic disk fore-/back-ground field stars, which are often located in the same region of the color magnitude diagram as blue straggler stars.

Methods. We analyze photometry taken from the literature of 3 open clusters of intermediate/old age rich in blue straggler stars, which are projected in the direction of the Perseus arm, and study their spatial distribution and the color magnitude diagram.

Results. As expected, we find that a large portion of the blue straggler population in these clusters are simply young field stars belonging to the spiral arm. This result has important consequences on the theories of the formation and statistics of blue straggler stars in different population environments: open clusters, globular clusters, or dwarf galaxies.

Conclusions. As previously emphasized by many authors, a detailed membership analysis is mandatory before comparing the blue straggler population in star clusters to theoretical models. Moreover, these sequences of young field stars (blue plumes) are potentially powerful tracers of Galactic structure and they require further consideration.
\end{abstract}

Key words. stars: blue stragglers - Galaxy: open clusters and associations: general - Galaxy: disk

\section{Introduction}

In the past few years, blue straggler stars (BSS) have received considerable attention. These bright stars appear above the turn off point (TO) in the color-magnitude diagram (CMD) of stellar populations, along the extension of the main sequence (MS) and are almost ubiquitous. In fact BSS have been detected in globular clusters (Sandage 1953; Ferraro et al. 2003), dwarf galaxies in the Local Group (Momany et al. 2007), and Galactic open clusters (OCs) (Ahumada \& Lapasset 2007; De Marchi et al. 2006; Xin et al. 2007). The formation mechanism of BSS in different environments is still being debated, and we refer the reader to the papers quoted above for all the details.

According to the recent analysis by Momany et al. (2007), Galactic open clusters, although being less populous than globular clusters and dwarf galaxies, seem to contain the highest percentage of BSS (see their Fig. 2). This appears to imply that the formation mechanism of BSS depends on the environment, since it is different in open and globular clusters and in dwarf spheroidal galaxies. However, open clusters are embedded in the Galactic disk and are more affected by field-star contamination than globulars. The amount of contamination depends on the cluster position and can only be quantified by a detailed membership analysis, which unfortunately is available for only

\footnotetext{
* On leave from Dipartimento di Astronomia, Università di Padova, Vicolo Osservatorio 2, 35122 Padova, Italy.
}

a handful of clusters (see Ahumada \& Lapasset 2007, for more details).

A related problem is in fact that blue sequences in the CMD of dwarf galaxies (the so-called blue plumes) are very similar to BSS sequences in open and globular clusters; but at the same time, they can be made of young stars, which are residuals of recent star formation episodes (Momany et al. 2007).

A remarkable case is Canis Major (CMa, Bellazzini et al. 2004), a putative dwarf galaxy immersed in the third quadrant of the Galactic disk. The prominent blue plume visible in CMDs has been considered to be composed of 1-2 Gyr old stars and/or BSS (Bellazzini et al. 2006) belonging to CMa.

However, in a series of papers (Carraro et al. 2005; Moitinho et al. 2006; Pandey et al. 2006), similar sequences have been observed in CMDs across the entire third Galactic quadrant, and it has been argued - based on a larger wavelength coverage - that they are composed of young stars belonging to previously poorly known spiral features. These different views highlight the delicacy of interpreting CMDs and the critical choice of photometric bands.

In this Note we investigate the BSS population in three OCs (NGC 7789, Berkeley 66, and Berkeley 70), located in the second Galactic quadrant, where the spiral structure is well known. We draw attention to the effect of field star contamination in cluster CMDs and to the need for detailed membership studies as the observational foundation for robust theoretical 


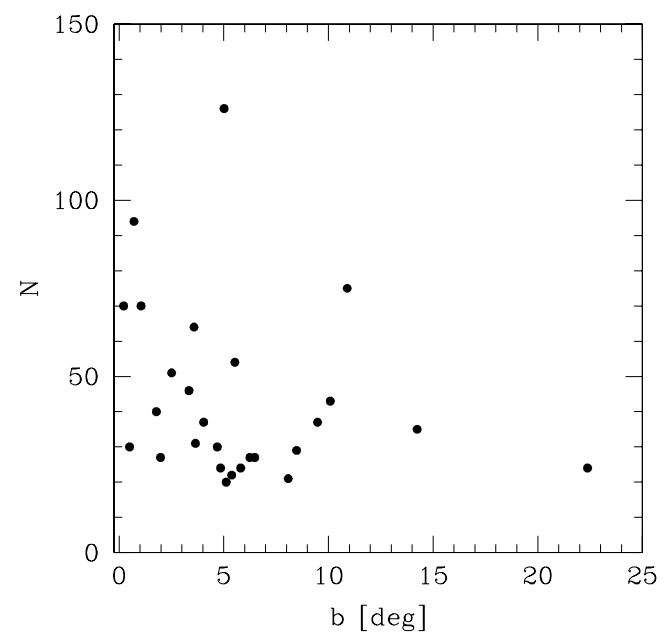

Fig. 1. Absolute number of BSS in the most BSS-rich open clusters (AL07) vs. their Galactic latitude.

investigations of BSS formation mechanisms. Moreover, we show that the blue sequences like those found in the third Galactic quadrant are also present in the second quadrant and, in most cases, can be ascribed to spiral features.

\section{The role of field star contamination}

Our starting point is the new release of the BSS catalog by Ahumada \& Lapasset (2007, hereinafter AL07). We opted to use this compilation among those at our disposal because it contains the most up-to-date list of BSS candidates in OCs. As the authors state, accurate membership studies based either on radial velocity or proper motion are unfortunately only available for a limited number of clusters; therefore, it is only possible to provide BSS candidates according to some less accurate criteria. That BSS stars may occupy the same locus as field stars in the CMD is the main difficulty in providing robust BSS statistics.

To illustrate how crucial this contamination is, we consider the star clusters with the largest absolute populations of BSS listed in Table 6 of AL07. We remove Ruprecht 46 from the sample, because - interestingly for the purpose of this Note - it has been demonstrated to be a chance alignment of field stars (Carraro \& Patat 1995); yet, this random enhancement of field stars leads the entries of clusters having the largest relative population of BSS (see Table 7 of AL07). In Fig. 1, we plot the 28 entries of Table 6 of AL07 as a function of Galactic latitude. Clearly, there is a trend toward having more BSS at lower Galactic latitudes, where field star contamination becomes greater.

Recently, Momany et al. (2007) have shown that field star contamination is the source of an artificially higher BSS fraction in the globular clusters NGC $6717\left(l=-11^{\circ}\right)$ and NGC 6838 $\left(l=-5^{\circ}\right)$ when compared to clusters of similar absolute magnitude but located at higher Galactic latitudes. The same seems to occur for Galactic open clusters. In the AL07 catalog - but also in all the other compilations (Xin et al. 2007; De Marchi et al. 2007) - M 67 and Berkeley 18 are two coeval and similar metallicity clusters; still, Berkeley 18 at $(l, b)=(163.63,+5.02)$ has 4 times more BSS than M 67 at $(l, b)=(215.69,+31.89)$.

\section{The spiral structure of the Milky Way}

The majority of OB type stars are located in spiral arms and form sequences in the CMD blurred by differential reddening and distance spread due to the patchy and irregular shape of the arms. Still, these sequences are prominent. In the past years we have provided evidence of such sequences in the fields of open clusters in the third Galactic quadrant (TGQ, Carraro et al. 2005; Moitinho et al. 2006), where no previously accepted indications of spiral arms were present. Similar results have been obtained by Pandey et al. (2006) for a sample of clusters in the 3GQ and second Galactic quadrant (SGQ).

We searched for spiral arm traces along the lines-ofsight of clusters listed in Table 6 of AL07 to understand whether BSS candidates could be mostly interpreted as young field stars. As an illustration, we consider 3 cases here: NGC 7789, Berkeley 66 and Berkeley 70, located at $(l, b)=$ $(115.53,-5.38),(139.43,+0.22)$, and $(166.89,+3.58)$, respectively. These are three OCs with high relative populations of BSS (AL07, Table 7) and that are well-separated in the SGQ. At odds with the case of the TGQ, these three clusters are located in the SGQ along directions where the presence of Perseus and of the Local arm are well-established (Russeil et al. 2007). Moreover, according to the maps of Burton (1985), one can detect HI emission in these directions due to the prominent Local arm, which surrounds the Sun, and to the more distant, detached Perseus arm.

\subsection{NGC 7789}

At $l=115^{\circ}$ (Galactic longitude of NGC 7789), the maps of Burton (1985) clearly indicate that the Perseus arm is detached from the Local arm and show HI emission down to at least $b=-6^{\circ}$. This implies that contamination from young stars in the Perseus arm can be important in the field of NGC 7789. In the upper panels of Fig. 2 we show the CMD of NGC 7789 (left panel) and of the surrounding field (right panel). In the lower panel we plot the stellar spatial distribution and illustrate the cluster region with a circle that is 14 arcmin wide (the innermost one). An annulus far from the cluster and with the same area as the clusters is indicated with the two outermost circles. Together with all the observed stars, we indicate the spatial distribution of the candidate BSS, as selected in each CMD. We adopted the same criteria as AL07 for the selection (see their Fig. 1): The solid isochrone and ZAMS are drawn using the fundamental parameters of NGC 7789 (see Table 1) to show the region of the CMD where BSS candidates are to be searched for. We are aware that for this specific cluster a number of BSS (22) have been found to be cluster members, indicated in Fig. 2. However, for the aim of this Note, what is actually relevant is the number of candidate BSS (lacking any membership analysis in most cases, all stars in the appropriate region of the CMD are routinely considered to be BSS candidates), so we did not remove the members BSS from the candidate sample. The first important fact to highlight is that candidate BSS are evenly distributed across the field. In the CMDs in NGC 7789 area (Fig. 2, upper panels), we recognize a vertical sequence right above the TO and an almost parallel, detached, bluer sequence. The BBS (see also Fig. 4 in AL07) do not follow closely any of these sequences, but are significantly spread in color.

While the sequence right above the TO is most probably caused by a mix of binary stars and nearby field stars, the bluer sequence resembles the blue plumes found either in dwarf galaxies (Momany et al. 2007), or in the background of open clusters 

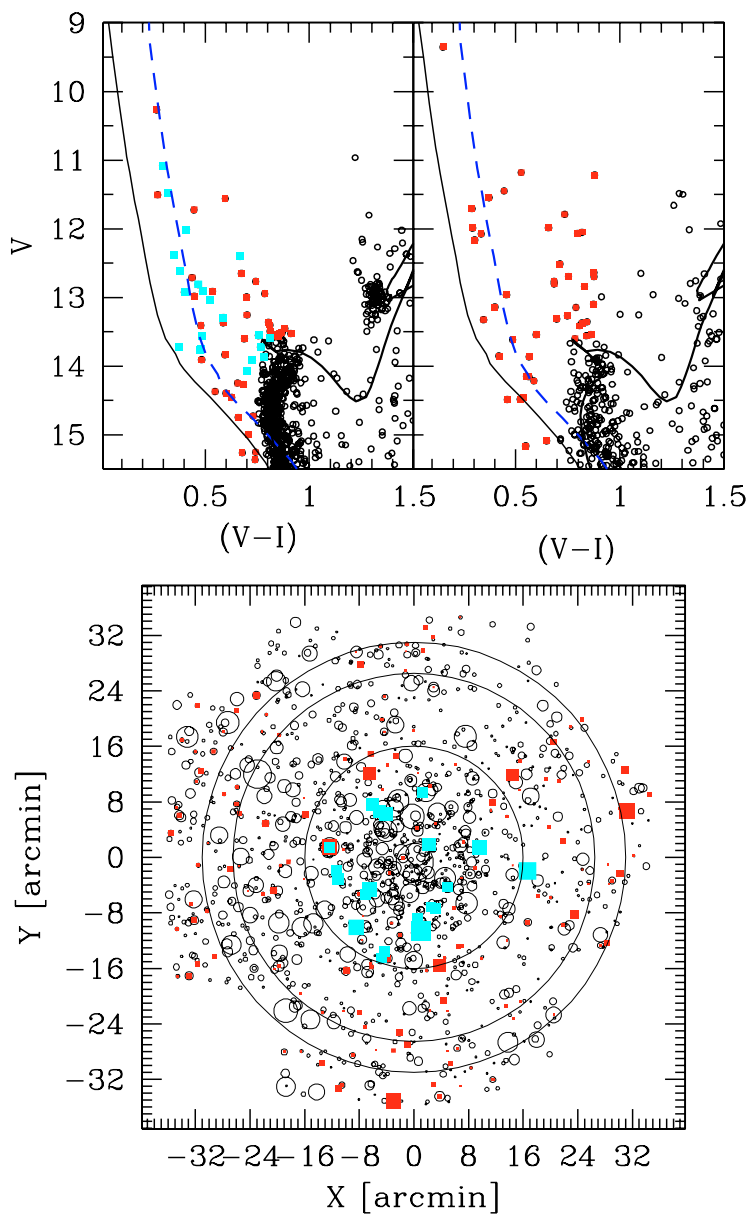

Fig. 2. Location in the CMD of NGC 7789 of BBS and field stars. In the upper left panel we show CMDs of NGC 7789 from Gim et al. (1998) for all stars brighter than $V \sim 15.50$ and within the cluster radius (see Table 1). In the upper right panel, the CMD of an equal area field is shown for comparison. The cluster and field area are indicated in the lower panel, where the field of NGC 7789 covered by Gim et al. (1998) photometry is shown. In this panel the innermost circle indicates the cluster area, while the two outermost circles illustrate the field selection. This annulus have been chosen to have the same area and at the same time to be as far as possible distant from the cluster, to avoid contamination from NGC 7789 external halo stars. In all the panels filled squares (in red in the electronic version) refer to BSS stars, according to AL07 criteria (see text). Different color filled squares (in cyan in the electronic version) are used for the BSS members of NGC 7789. In the CMDs, the two solid lines are an isochrone and a ZAMS drawn for the value of the fundamental parameters of NGC 7789 (see Table 1). The dashed line (blue in the electronic version) has been drawn for the typical distance of the Perseus arm in the NGC 7789 direction.

in the 3GQ (Carraro et al. 2005). The bulk of these blue stars do not follow the solid ZAMS (compatible with stars at the same distance and reddening of NGC 7789), but lie redward. We suggest that this is a sequence of more distant and reddened young stars, similar to a blue plume. The dashed ZAMS (blue when printed in color) we super-imposed on it would in fact imply a distance of about $3 \mathrm{kpc}$, significantly more than the distance of NGC 7789 (less than $2 \mathrm{kpc}$ ). Along the blue plume we count about 34 stars. From AL07 we know that NGC 7789 harbors 22 BSS which are almost entirely concentrated within the cluster radius (see the bottom panel map). However, only a fraction of them (13) lie close to this blue sequence.
Table 1. Fundamental parameters of the clusters under study, taken from WEBDA.

\begin{tabular}{ccccccc}
\hline \hline Name & $\begin{array}{c}l \\
\mathrm{deg}\end{array}$ & $\begin{array}{c}b \\
\mathrm{deg}\end{array}$ & $\begin{array}{c}\text { Radius } \\
\text { arcmin }\end{array}$ & $(m-M)_{V}$ & $E(V-I)$ & $\begin{array}{c}\text { age } \\
\mathrm{Gyr}\end{array}$ \\
\hline NGC 7789 & 115.53 & -5.38 & 14.0 & 12.5 & 0.27 & 1.7 \\
Berkeley 66 & 139.43 & +0.22 & 2.0 & 17.5 & 1.55 & 5.0 \\
Berkeley 70 & 166.89 & +3.58 & 2.5 & 14.6 & 0.60 & 4.7 \\
\hline
\end{tabular}

We conclude that a substantial fraction of the stars in the blue plume are young field stars located in the southern tail of the Perseus arm along the cluster's line of sight, as is also evident from the BSS candidates position in the field CMD (upper right panel). This result is supported by the HI radial velocity in the range of $-65:-75 \mathrm{~km} \mathrm{~s}^{-1}$ (Burton 1985), somewhat higher than NGC $7789\left(-54.9 \pm 0.12 \mathrm{~km} \mathrm{~s}^{-1}\right.$, Gim et al. 1998). Moreover, the NGC 7789 isochrone distance is in fact shorter than $2.0 \mathrm{kpc}$, while the distance to the HII regions associated to the arm (S163, S164, S166, and S170, for instance) at the same longitude of the cluster are greater (2.5-2.9 kpc, Russeil et al. 2007), and are compatible with the blue plume.

\subsection{Berkeley 66}

This is a distant open cluster, located well beyond the Perseus arm, but very close to the plane, and very reddened for that reason (Phelps \& Janes 1996; Guarnieri \& Carraro 1997; Villanova et al. 2005). It represents an interesting case since it lies along the line of sight where Xu et al. (2006) estimate the distance to the Perseus arm to be about $2 \mathrm{kpc}$. In Fig. 3 we show the CMDs of Berkeley 66. The photometry is taken from Phelps \& Janes (1996), who covered a 5'.1 × 5'.1 field around the cluster's nominal center and found that it has a radius smaller than 2 arcmin. This field of view is small, and therefore we do not expect to find many stars associated to the Perseus arm, which lies much closer than the cluster.

In the left panel we consider only stars within the nominal cluster radius ( 2 arcmin, see Table 1 ), as defined by the circle in the lower panel map. We superimposed an isochrone for the values of the basic parameters as in Table 1. A ZAMS for this reddening and distance modulus is also plotted with the same symbols to guide the eye and indicate the region where BSS are to be searched for, according to the AL07 criteria. The same representation is used in the field CMD (upper right panel), which contains all the stars outside the cluster area. This field - limited by the outermost circle in the map - has the same area as the cluster region. The spatial distribution of BSS candidates in the cluster and field is show in the bottom panel, where the area covered by Phelps \& Janes photometry is shown.

Within the cluster radius (2') we count 63 BSS candidates (AL07 indicate 70, but possibly used a different radius). All stars 2.5 mag brighter than the TO are taken as BSS candidates. However, looking at the field CMD, the color and magnitude distribution of BSS candidates and the field stars are similar. In fact, we count 68 BSS candidates in the field. It is therefore likely that a significant number of BSS candidates are just field stars.

Although located behind the Perseus arm, no obvious traces of a tight young star sequence associated to the arm (blue plume) are found in the field of Berkeley 66. This is most probably due to the small field of view of the observations, together with the highly irregular absorption and/or to the patchy structure of the arm. 

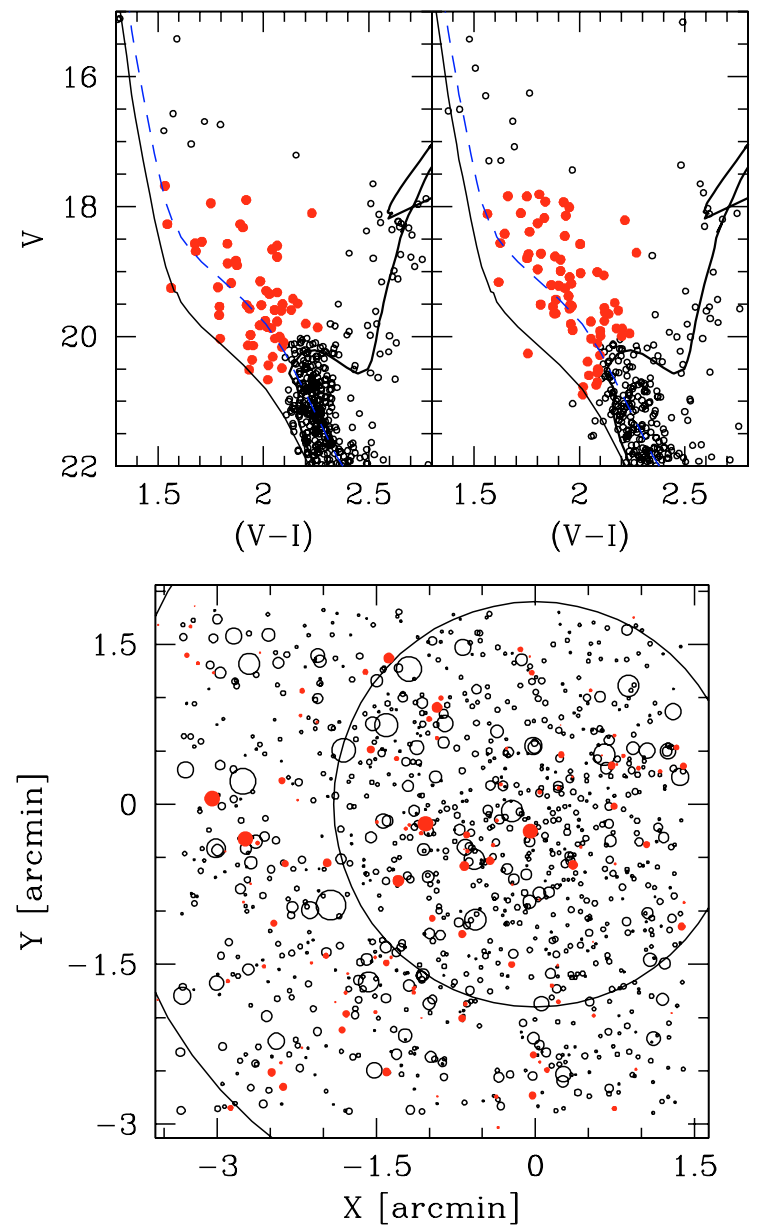

Fig. 3. Upper panels: CMD of Berkeley 66 for cluster (left) and field (right) regions, as defined in the lower panel, where the innermost circle is drawn by adopting 2.0 arcmin for the cluster radius (see Table 1). The region outside the cluster area and limited by the outermost circle has the same area as the cluster region. Solid symbols (red in the electronic version) indicate BSS candidates. The solid lines are a ZAMS and an isochrone for the most accepted values of the cluster fundamental parameters (as in Table 1), whereas the dashed line (blue in the electronic version) indicates the expected location of young stars at the distance of the Perseus arm.

\subsection{Berkeley 70}

Berkeley 70 is another old star cluster reported to be very rich in BSS. AL07 list 64 BSS candidates, virtually all the stars above the TO. Although Berkeley 70 is relatively high above the Galactic plane $\left(b=+3.6^{\circ}, Z\right.$ approximately $\left.250 \mathrm{pc}\right)$, Burton (1985) detected considerable $\mathrm{HI}$ emission in its direction, which indicates that the Perseus arm is significantly thick, reaching up to $b=+8^{\circ}$. We take the photometry of Berkeley 70 from Ann et al. (2002), who studied of a $5 ! 8 \times 5 ! 8$ field centered on the cluster. The CMD is presented in Fig. 4 for the cluster (upper left panel) aqnd field (upper right panel) region, together with the spatial distribution of all the stars and the candidate BSS in the lower panel map. Here the innermost circle corresponds to the cluster radius ( $\sim 2.5$ arcmin), whereas the region outside the cluster area and limited by the outermost circle identifies an equal-area stellar field.

In the CMDs we superimposed an isochrone and a ZAMS using the values from Table 1, and marked BSS candidates (following AL07). The upper part of the CMD is characterized by a number of blue stars close to the prolongation of the cluster's
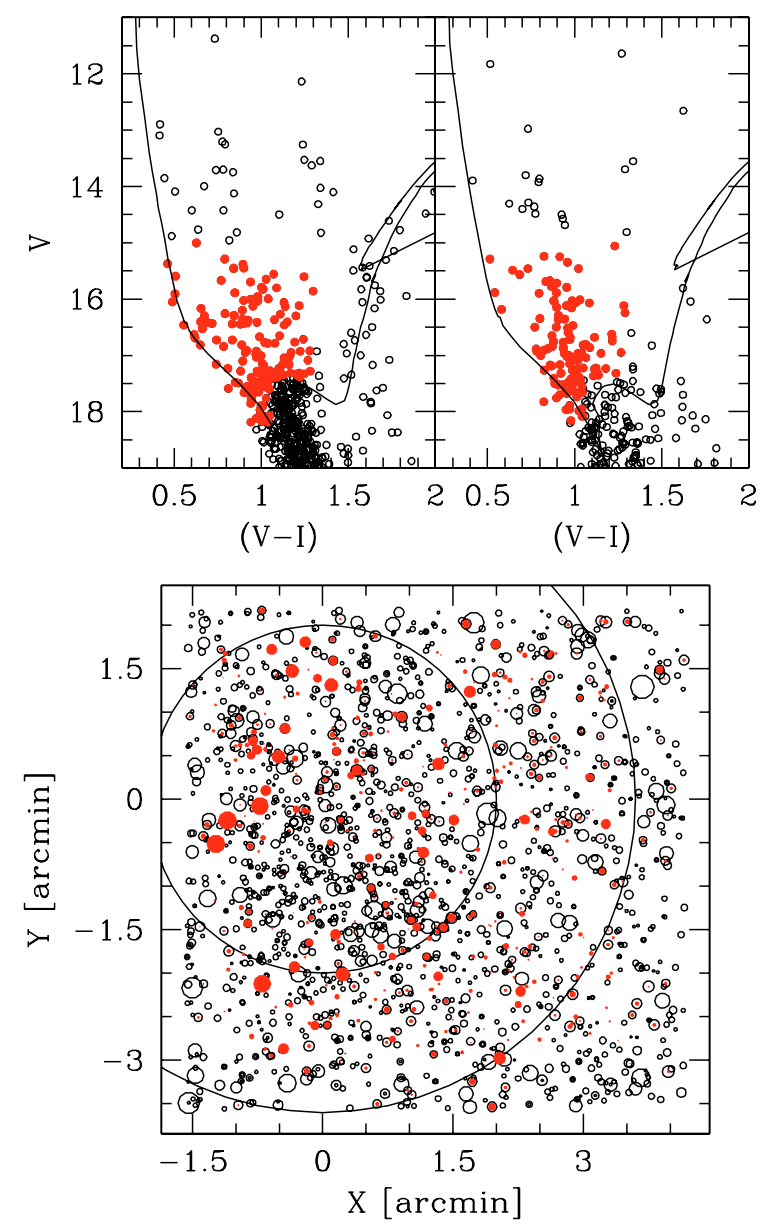

Fig. 4. Upper panels: CMD of Berkeley 70 for cluster (left) and field (right) regions, as defined in the lower panel, where the innermost circle is drawn by adopting 2.5 arcmin for the cluster radius (see Table 1). The region outside the cluster area and limited by the outermost circle has the same area of the cluster region. Solid symbols (red in the electronic version) indicate BSS candidates. The solid lines are a ZAMS and an isochrone for the most accepted values of the cluster fundamental parameters (as in Table 1).

ZAMS and another more scattered sequence right above the TO. The first of the two is a sequence of young stars at about the same distance of the cluster $(\sim 4.0 \mathrm{kpc})$, similar to the distance to the Perseus arm in this direction (Russeil et al. 2007), so that a fraction of these blue stars are likely to belong to the arm. Therefore, for the specific case of Berkeley 70, the Perseus arm young stars have roughly the same distance as the cluster, and for this reason we do not plot any other ZAMS.

As a further confirmation of their field star nature, these stars are evenly distributed across the observed field and - according to the cluster radius ( $2.5 \mathrm{arcmin})$ - only a fraction of them lie within the cluster. The other, redder, and more scattered sequence is probably due to contamination of field stars near the Sun, including stars in the Local arm. Beside, we note that other bright stars (brighter than the upper limit for BSS candidates according to AL07) lie close to this ZAMS, emphasizing their nature as young field stars associated with the arm. Also in this case, the number and distribution of BSS candidates in the cluster and field CMDs are very similar. We therefore conclude that the distribution of young blue stars in Berkeley 70 is that expected from the location of the cluster, right above and at the same distance as the Perseus arm. 


\section{Conclusions}

The aim of this Note was twofold. On one side, we wanted to stress once again how derived statistics of BSS stars in Galactic OCs is crucial for properly accounting for field star contamination. Specifically, this paper addresses the effect of contamination introduced by the early type stellar component of spiral arms. Trends like the anti-correlation between absolute magnitude and BSS frequency (Piotto et al. 2004) must be reconsidered in the light of accurate radial velocity or proper motion-based memberships. Only in this way will the derived BSS population in OCs be statistically meaningful. We are in the process of starting such an observational effort for a number of selected open clusters.

On the other hand, we have shown how the bulk of the stars populating the upper part of three representative open clusters (NGC 7789, Berkeley 66, and Berkeley 70) in the SGQ is mostly dominated by young stars located near the Sun - in the Local arm - and in the Perseus arm, which lies in front of (for Berkeley 66 and Berkeley 70), or beyond (for NGC 7789) these clusters. This was possible since we already know the existence, distance, and location of the Perseus arm. This confirms that similar sequences found in the CMDs of stellar fields in other regions of the Galaxy can be used as spiral-arm tracers.

Acknowledgements. This study made use of SIMBAD and WEBDA. A.M. acknowledges support from FCT (Portugal) through grant PDCT/CTEAST/57128/2004. R.A.V. acknowledges the financial support from the
CONICET PIP 5970. We thank the anonymous referee for her/his suggestions, which helped us to improve the paper's presentation.

\section{References}

Ahumada, J. A., \& Lapasset, E. 2007, A\&A, 463, 789

Ann, H. B., Lee, S. H., Sung, H., et al. 2002, AJ, 123, 905

Bellazzini, M., Ibata, R., Martin, N., Irwin, M. J., \& Lewis, G. F. 2004, MNRAS, 354,1263

Bellazzini, M., Ibata, R., Martin, N., et al. 2006, MNRAS, 366, 865

Burton, W. B. 1985, A\&AS, 62, 365

Carraro, G., \& Patat, F. 1995, MNRAS, 276, 563

Carraro, G., Vázquez, R. A., Moitinho, A., \& Baume, G. 2005, ApJ, 630, 153

Dias, W. S., Alessi, B. S., Moitinho, A., \& Lepine, J. R. D. 2002, A\&A, 389, 871

Girardi, L., Bressan, A., Bertelli, G., \& Chiosi, C. 2000, A\&AS, 141, 371

De Marchi, F., De Angeli, F., Piotto, G., Carraro, G., \& Davies, N. B. 2006, A\&A, 459, 489

Ferraro, F. R., Sills, A., Rood, R. T., Paltrinieri, B., \& Buonanno, R. 2003, ApJ, 588,464

Gim, M., Vandenberg, D. A., Stetson, P. B., Hesser, J. E., \& Zurek, D. R. 1998, PASP, 110,1318

Guarnieri, M. D., \& Carraro, G. 1997, A\&AS, 121, 451

Moitinho, A., Vázquez, R. A., Carraro, G., et al. 2006, MNRAS, 368, L77

Momany, Y., Held, E. V., Saviane, I., et al. 2007, A\&A, 468, 973

Pandey, A. K., Sharma, S., \& Ogura, K. 2006, MNRAS, 373, 255

Phelps, R. L., \& Janes, K. A. 1996, AJ, 111, 1604

Piotto, G., De Angeli, F., King, I. R., et al. 2004, ApJ, 604, L109

Russeil, D., Adami, C., \& Georgelin, Y. M. 2007, A\&A, 470, 161

Sandage, A. R. 1953, AJ, 58, 161

Schlegel, D. J., Finkbeiner, D. P., \& Davis, M. 1998, ApJ, 500, 525

Vázquez, R. A., May, J., Carraro, G., et al. 2008, ApJ, 672, 930

Villanova, S., Carraro, G., Bresolin, F., \& Patat, F. 2005, AJ, 130, 652

Xin, Y., Deng, L., \& Han, Z. W. 2007, ApJ, 660, 319

Xu, Y., Reid, M. J., Zheng, X. W., \& Menten, K. M. 2006, Science, 311, 54 